\title{
The effects of stress on singing voice accuracy
}

\author{
Larrouy-Maestri, P. \& Morsomme, D. \\ Logopédie de la Voix, Department of psychology: Cognition and Behavior, \\ University of Liège, Belgium
}

The results of this study were accepted and presented as a poster presentation at the Voice Foundation's $41^{\text {st }}$ Annual Symposium (Philadelphia, May 31, 2012).

Correspondence concerning this article should be addressed to Pauline Larrouy-Maestri, Logopédie de la voix, Département de Psychologie, Université de Liège, B-38, Rue de l'Aunaie, 30, 4000 Liège, Belgium. Phone: +32486538413.

E-mail may be sent to pauline.larrouy@ulg.ac.be. 


\begin{abstract}
Objective: The quality of a music performance can be lessened or enhanced if the performer experiences stressful conditions. In addition, the quality of a sung performance requires control of the fundamental frequency of the voice, which is particularly sensitive to stress. The present study aimed to clarify the effects of stress on singing voice accuracy.

Methods: Thirty-one music students were recorded in a stressful condition (i.e., a music examination) and a non-stressful condition. Two groups were defined according to the challenge level of the music examination (first and second music levels). Measurements were made by self-reported state anxiety (CSAI-2R questionnaire) and by observing heart rate activity (electrocardiogram) during each performance. In addition, the vocal accuracy of the sung performances was objectively analyzed.
\end{abstract}

Results: As expected, state anxiety and heart rate were significantly higher on the day of the music examination than in the non-stressful condition for all the music students. However, the effect of stress was positive for the first-year students but negative for the second-year students, for whom the music examination was particularly challenging. In addition, highly significant correlations were found between the intensity of cognitive symptoms and the vocal accuracy criteria.

Discussion: This study highlights the contrasting effects of stress on singing voice accuracy but also the need to consider the challenge level and perception of the symptoms in experimental and pedagogical settings.

\title{
Running head
}

Stress and singing voice

\section{Keywords}

singing voice; vocal accuracy; anxiety; stage fright; stress; music students 


\section{Introduction}

Singing voice accuracy depends on variations in fundamental frequency $\left(\mathrm{f}_{0}\right)$ during a sung performance. ${ }^{1-6}$ In a melodic context, the relation between the tones (i.e., musical intervals) and the tonal center of the melody must be respected if the performer is to be considered as singing in tune. Indeed, two kinds of errors are considered in the judgment of vocal accuracy: ${ }^{7}$ the precision of the musical intervals and the respect of the tonality of the tune. The former is assessed by measuring the difference between each interval produced and the theoretical interval given by the musical score. ${ }^{1-7}$ The latter is based on the consistency of the harmonically important notes performed in the course of the tune. ${ }^{4}$ In order to obtain low scores for these criteria (i.e., a high accuracy level), multiple components are required. ${ }^{8-10}$ Indeed, the perceptual, motor, sensorimotor and memory components must all function properly to control the $\mathrm{f}_{0}$ variations in the sung performance and thus to sing in tune. Note also that there is a relationship between singing voice accuracy and the tempo of the performance for both untrained singers (the slower, the more accurate) $)^{2,3}$ and professional singers (the faster, the more accurate). ${ }^{4}$

Most studies of singing voice accuracy have observed untrained singers in an experimental context but this condition does not reflect stage performance, which can be stressful. For instance, performing in public or under pressure (e.g., in an examination) is reported to be stressful for musicians, ${ }^{11}$ particularly in a Western classical context. ${ }^{12,13}$ Some authors use the terms "stage fright" and "music performance anxiety" interchangeably to describe the consequences of stress, ${ }^{14-16}$ whereas others distinguish these terms depending on the severity of the stress level. ${ }^{17}$ The somatic and cognitive symptoms induced by a stressful situation and their interpretation by the performer can be observed by using questionnaires. For example, the performer may report perceived physiological 
arousal or negative thoughts and interpret them as facilitative or debilitative. Objective measurements of physiological manifestations of stress can also be observed. ${ }^{11,18-25}$ Indeed, the heart rate is dramatically higher during a stressful condition such as a competition than during rehearsals. ${ }^{18-25}$

As heart rate variations have a significant influence on the $\mathrm{f}_{0}$ of the speaking voice and of sustained voice productions, ${ }^{26,27}$ we hypothesize that a stressful condition should have an impact on vocal accuracy. However, this impact could be positive or negative. Studies of the speaking voice have shown that $f_{0}$ increases with stress ${ }^{27-30}$ but vocal accuracy in a melodic context depends on the relation between the notes and cannot be limited to the increase in $\mathrm{f}_{0}$. An objective analysis of the vocal accuracy criteria would be necessary to observe the positive or negative effect of stress on the accuracy of a vocal performance. More generally, the relationship between physiological arousal and performance has been investigated since the inverted-U hypothesis of Yerkes and Dodson. ${ }^{31}$ According to Yerkes and Dodson's law (1908), performance increases with physiological or mental arousal and decreases when the level of arousal becomes too high. More recently, Yoshie et al. ${ }^{32}$ reported a negative change in performance quality among pianists when they performed in a stressful condition compared to without pressure. Their results did not confirm the findings of previous studies that reported no significant differences in performance quality between evaluative and non-evaluative conditions, ${ }^{20,24}$ or even an improvement under jury conditions. ${ }^{22}$ These contrasting findings may be explained by a lack of control over stress, in terms of level or type. A combination of physiological and psychological measurements is thus necessary to evaluate the stress manifestations. In addition, the difference in results could depend on the challenge level of the stressful condition for the participants. In past studies, college-level music students, ${ }^{22}$ 
competent, ${ }^{20}$ skilled amateurs, ${ }^{24}$ and highly trained pianists ${ }^{32}$ were observed in non-stressful and stressful conditions but the implications of the context of the musical performance (i.e., crucial examination or just part of the musical training) were not examined. ${ }^{20,22,24,32}$

In order to clarify the effects of stress on sung performances, the present study observed the physiological and psychological manifestations induced by a stressful situation and their effects on singing voice accuracy in music students at two levels (i.e., low and high challenge levels). For this purpose, we analyzed the sung performances of first- and second-year students in a stressful (music examination) and a non-stressful condition. Our aim was (a) to confirm that a music examination represents a stressful condition through the observation of heart rate activity and self-reported state anxiety, (b) to observe the changes in the vocal performances by objectively analyzing the vocal accuracy of each recording, and (c) to compare two music levels (low versus high challenge levels) in terms of the changes in stress and vocal accuracy.

\section{Methods}

\section{Participants}

Thirty-one music students (19 men, 12 women) from the Royal Conservatories of Belgium made up the sample. Their age ranged from 14 to $24(M=19.29)$. In order to be accepted into these institutions, music students have a singing audition, in addition to their instrumental audition. Their ability to read and sing musical scores was thus assured. The solfeggio classes are held during the first two years of the music program (i.e., first and second levels). Note that the two solfeggio examinations are similar, with the same jury, but that the second-level examination is more important as it represents the end of the solfeggio classes, which is a condition for continuing in the music program. Eighteen 
participants were in their first year (aged from 14 to 24 years old, $M=19.22$ ) and 13 were in their second year (aged from 16 to 23 years old, $M=19.38$ ). Participants were instructed about the goal of the study, received an information form, signed the consent form and knew that they could stop their participation at any time.

\section{Procedure}

The collaboration with the Royal Conservatories and the support of the teaching staff meant we could have students learn a melody during the solfeggio classes, once a week for two months (Learning phase in Figure 1) before the first recording. The performance was then recorded several times, with three weeks between sessions (see Figure 1). "Habituation" corresponds to a condition which allowed music students to get acquainted with the protocol, the experimenter and the equipment. Participants were instructed to sing the melody they had learned (Figure 2) a cappella, after hearing the first note played on a piano by the experimenter. As indicated on the score, they had to perform with repeats, at a tempo of 80 beats per minute. The melody was sung with the syllable /no/ in order to avoid hesitations due to the spelling of the words and to provide a clear auditory signal to segment and analyze. In the stressful condition, the participants had to sing the melody in front of a jury composed of four music experts: two solfeggio teachers, one musician with experience in solfeggio evaluation and the pianist who accompanied the singing examination. The melody was performed at the beginning of the solfeggio examination. In the non-stressful condition, participants sang the melody in front of the experimenter in a quiet room at the Conservatories. This condition was similar to the "habituation" condition.

Insert Figure 1 about here 


\section{Material}

\section{Audio data}

The melody the students learned (Figure 2) was inspired by the popular song "Happy Birthday" and created in order to use the criteria and analysis tools validated by Larrouy-Maestri and Morsomme. ${ }^{4}$ Audio was picked up by a Sennheiser HS2 head-worn microphone (Wedemark, Germany) positioned at a constant distance of $2 \mathrm{~cm}$ from the right corner of the mouth and recorded on a Marantz PMD67 recorder (Kanagawa, Japan). The sung performances were digitalized with a sampling frequency of $44.1 \mathrm{kHz}$ and a 16-bit resolution.

\section{Insert Figure 2 about here}

\section{Stress level evaluation}

Objective measurements: electrocardiogram. Participants wore a finger sensor, linked to a DATEX OHMEDA S/5 monitor (Madison, USA) connected to a ThinkPark IBM laptop. The heart rate (beats per minute) was recorded in the habituation, stressful and non-stressful conditions (Figure 1). In order to observe the changes in heart rate between the two conditions, we selected the non-stressful condition as a baseline and observed the difference between the stressful condition and the baseline for each participant.

Subjective measurements: questionnaire. Cognitive and somatic symptoms were assessed just before the examination (i.e., the stressful condition) and in the non-stressful condition (Figure 1), with the Competitive State Anxiety Inventory - 2 Revised (CSAI-2R) questionnaire, ${ }^{33}$ translated and validated in French. ${ }^{34}$ Although this material was designed to assess state anxiety in sports competitions, it has also been used for music performance. ${ }^{13,25}$ Because of the limited time available for students to fill in the 
questionnaire, we selected 11 items, which correspond to the two subscales designed to investigate somatic and cognitive symptoms. Participants had to rate the intensity of each symptom on a scale of 1 (not at all) to 4 (very much) at the time of the performance. Thus, the questionnaire was administered immediately before each sung performance. For the direction scale, the participants rated on a scale ranging from -3 to +3 the degree to which the experienced intensity of each symptom was either debilitative or facilitative, with 0 indicating that it was considered to be unimportant. A positive score represents a state of facilitation and a negative score a state of debilitation. In order to observe the changes in somatic and cognitive symptoms between the different conditions, we selected the nonstressful condition as the baseline and observed the difference between the stressful condition and the baseline for each participant.

\section{Evaluation of sung performances}

\section{Acoustical analyses}

The data were processed semi-automatically in two stages on a MacBook Pro (Mac OS X, Version 10.6.5). Analyses were done with AudioSculpt 2.9.4v3 and OpenMusic 6.3 software (IRCAM, Paris, France) using a Short-Time Fourier Transform (STFT) analysis. Following the analytical procedure of Larrouy-Maestri and Morsomme, ${ }^{4}$ markers were manually placed on the spectrogram in order to avoid the attacks and the glides between notes and minimize the influence of musical interpretation of the melody. Then, the average $\mathrm{f}_{0}$ was automatically calculated and converted into cents $(1 / 2$ tone $=100$ cents $)$ for the 18 notes selected from the melody (Figure 2). 


\section{Vocal accuracy criteria}

Concerning the intonation of the melody, the measurements were based on $\mathrm{f}_{0}$ differences between the tones in an equal temperament (i.e., equality of the semitones). For each sung performance, the interval deviation and tonal center deviation were examined according to the procedure described by Larrouy-Maestri and Morsomme. ${ }^{4}$ For the interval deviation criterion, each interval was computed by subtracting the $\mathrm{f}_{0}$ of the adjacent notes and comparing the values to the theoretical ones (i.e., on the basis of the musical notation). The final score corresponds to the mean of the deviations between the performed intervals and the theoretical ones. To determine the tonal center deviation criterion, the intervals performed between eight notes, which convey important information about the tonality of the melody (i.e., notes 1, 4, 7, 14, 15, 16, 17, 18 in Figure 2), were compared to the theoretical value given by the musical notation. As for the interval deviation criterion, the final score is measured in cents. Note that a small deviation reflected good interval precision and good respect for the tonal center of the melody. In order to observe the differences between vocal accuracy in the different conditions, we selected the nonstressful condition as the baseline and observed the difference between the stressful condition and the baseline for each participant.

\section{Teтpo}

The mean tempo was computed on the basis of the length of each performance and the number of beats of the melody (beats per minute).

\section{Statistical analyses}

Given the small number of participants at each music level, non-parametric tests were chosen to compare the two conditions (stressful and non-stressful). A Wilcoxon 
signed-rank test was used to assess differences in the variables under study (physiological, psychological and vocal accuracy) between the two conditions. This non-parametric test was run for each music level separately. Note that three participants performed the melody with contour errors (the direction of the intervals was not correct). As we focused on singing voice accuracy, these three participants were removed from the statistical analyses. Therefore, we had 16 participants at the first level and 12 at the second level. In order to observe the changes in the variables (physiological, psychological and vocal accuracy) between the non-stressful and stressful conditions, we selected the non-stressful condition as the baseline and observed the differences between the stressful condition and the baseline for each participant. A Mann-Whitney U test was run to look for a significant difference in the evolution of these variables between the two groups of participants.

Finally, Spearman correlation matrices were computed for each music level, in order to evaluate the relationships between the changes in stress manifestations (heart rate, intensity and direction of cognitive and somatic symptoms) and those in vocal parameters (tempo, interval deviation and tonal center deviation).

\section{Results}

\section{Evaluation of stress level}

Table 1 shows the descriptive statistics of the stress measurements (heart rate, CSAI-2R questionnaire) separately for each music level.

Insert Table 1 about here

As can be seen in Table 1, the objective and subjective measurements of stress were higher on the day of the examination (i.e., stressful condition) than in the non-stressful condition. 
Insert Table 2 about here

For each music level, the Wilcoxon signed-rank test showed statistically significant differences in the heart rate measurement, as well as on the questionnaire (intensity of the somatic symptoms, intensity and direction of the cognitive symptoms). As Table 2 shows, the direction of somatic symptoms was not statistically different between the stressful and the non-stressful conditions for participants at the first music level.

Insert Table 3 about here

The changes between the non-stressful and stressful conditions for each music level are summarized in Table 3, together with the results of the Mann-Whitney $U$ test and the pvalues. No statistically significant difference occurred between the first and second music levels, except for the direction of the somatic symptoms. For the second-year students, the change in the perception of somatic symptoms between the non-stressful and stressful conditions was greater and more negative than for the first-year students.

\section{Evaluation of sung performances}

Interval deviation criterion. As can be seen in Figure 3, the first-year students performed better in the stressful condition $(M=17.05, S E=1.45)$ than in the non-stressful condition $(M=28.63, S E=5.87)$. The Wilcoxon signed-rank test confirmed this difference $(z=-2.36, p=.019)$. For the second-year students, no significant difference appeared between the stressful $(M=39.35, S E=8.54)$ and non-stressful $(M=27.53, S E=8.14)$ conditions for this criterion $(z=-1.18, p=.24)$.

Insert Figure 3 about here

Tonal center criterion. As can be seen in Figure 4, the second-year students 
performed better in the non-stressful condition $(M=19.67, S E=3.17)$ than in the stressful condition $(M=40.61, S E=11.39)$. The Wilcoxon test confirmed this difference $(z=-2.59$, $p=.01)$. In the case of the first-year students, no significant difference appeared between the stressful $(M=20.06, S E=2.45)$ and non-stressful $(M=38.65, S E=11.33)$ conditions for this criterion $(z=-0.74, p=.46)$.

Insert Figure 4 about here

The different profiles of the music students, according to their level, were confirmed with the Mann-Whitney U test. Indeed, the changes between the non-stressful and stressful conditions differed according to the music level for the interval deviation criterion $(U=$ $50.50, p=.035)$ and the tonal center criterion $(U=48.00, p=.026)$. Whereas the stressful condition was positive for the first-year students in terms of the interval deviation criterion, this condition was negative for the second-year students' performance, as measured by the tonal center deviation criterion.

\section{Relationships between stress level and vocal accuracy}

For each variable under study, we computed the change between the stressful condition and the baseline (i.e., non-stressful condition) for each participant and observed the relationships between the variables. For each music level, no correlation was found between the objective measurement of stress (i.e., heart rate) and singing voice accuracy (interval deviation and tonal center deviation). For students at the first music level, significant positive correlations were found between the interval deviation criterion and the intensity of cognitive symptoms $(r(16)=.520, p=.039)$, and also the direction of these symptoms $(r(16)=.608, p=.013)$. In other words, the more intense the cognitive symptoms were, and the more negatively they were interpreted, the worse the precision of 
the intervals was along the melody. Note that no significant correlation was found between the interval deviation criterion and the intensity of somatic symptoms $(r(16)=.297, p=$ $.264)$ or the direction of these symptoms $(r(16)=.438, p=.089)$. For students at the second music level, a significant correlation was found between the tonal center deviation criterion and the intensity of cognitive symptoms $(r(12)=.773, p=.003)$. For these students, the more intense the cognitive symptoms were, the worse their respect of the tonal center became. However, there was no significant relationship between the interpretation of cognitive symptoms (facilitative or debilitative) and vocal accuracy in this group of music students $(r(12)=.452, p=.140)$. Note that there was no significant correlation between the tonal center deviation criterion and the intensity of somatic symptoms $(r(12)=.340, p=$ $.279)$ or the direction of these symptoms $(r(12)=.218, p=.495)$. Concerning tempo, there was no significant correlation with stress measurements or vocal accuracy, at either music level.

\section{Discussion}

In order to observe the effects, positive or negative, of stress on singing voice accuracy, we analyzed the sung performances of students at two music levels in a stressful and a non-stressful condition. These results have both pedagogical and experimental implications.

\section{Changes in stress level}

Our results confirmed objectively that musicians are more likely to feel anxious in conditions in which they are being evaluated. ${ }^{11}$ As expected, the heart rate and the intensity of cognitive and somatic symptoms were significantly higher in the stressful condition than 
in the non-stressful situation, for students at both music levels. In addition, the cognitive and somatic symptoms were experienced as more negative in the stressful condition for both groups. However, members of the two groups differ significantly in the manner in which they interpret their somatic symptoms. In fact, this change was significantly higher for the second-year students. Note that the solfeggio classes are given during the first two years of the music program at the Royal Conservatories of Belgium. The experimental conditions were similar for students at both music levels (i.e., same musical score, same habituation, and same jury) and the main difference concerned the level of the challenge. Indeed, whereas second-year students must pass this examination in order to continue their musical education, the first-year students have the possibility of continuing their education regardless of their results on the first examination. This study revealed that the heart rate of first- and second-year students was faster during the music examination than during the non-stressful condition, with a similar evolution between the two groups. However, the difference observed in the interpretation of somatic symptoms between the two groups (larger negative evolution for the second-year students) could be attributed to the challenge level of the situation. In addition to this explanation, one can hypothesize that the kind of stress perceived by the first- and second-year students differs. It would therefore be interesting to compare different kinds of stressful situations (e.g., concerts, competitions). Also, we focused on the physiological and psychological manifestations induced by a music examination, but these observations would have to be completed in future researches. For example, the self-confidence subscale of the CSAI-2R or trait anxiety questionnaires $^{35,36}$ could be proposed to develop the findings of the present study. However, the present experimental design provides a pertinent and ecological context for examining the effects of stress on the quality of a music performance. In 
addition, the results regarding the interpretation of somatic symptoms confirmed the relevance of taking challenge level into account in the observation of differences in singing voice accuracy.

\section{Changes in singing voice accuracy}

Two important vocal accuracy criteria were observed for the participants whose performance respected the melodic contour $(\mathrm{n}=28)$. Previous studies highlighted the impact of stress on $\mathrm{f}_{0}$ in the speaking voice ${ }^{27-30}$ and on the quality of music

performance. ${ }^{20,22,24,32,37}$ Because vocal accuracy is based on $\mathrm{f}_{0}$ variations and must be respected in a musical performance, it is not surprising that stress has an impact on vocal accuracy. However, the effect of stress was different depending on the music level of the participants: positive for the first-year students (i.e., greater interval precision under stress) and negative for the second-year students (i.e. tonal center less respected under stress). The results also pointed out the relationship between vocal accuracy and the psychological manifestations of stress. Interestingly, no correlation was found between the changes in heart rate or somatic symptoms and the quality of the sung performances. However, the precision of the intervals was linked with the intensity and direction of the cognitive symptoms of the first-year students whereas the intensity of these symptoms was linked to the tonal center criterion for the second-year students. For students at each music level, high-intensity cognitive symptoms had a negative impact on their vocal accuracy. These findings confirm that the relationship hypothesized by Yerkes and Dodson ${ }^{31}$ cannot be limited to the observation of physiological manifestations. Note that these manifestations might not be strong enough to have consequences for the vocal quality. We therefore encourage future researchers to induce more stress, for example, with the presence of a 
larger jury or audience and/or more difficult musical material in order to confirm that physiological manifestations of stress are not linked to vocal accuracy. In addition to the psychological manifestations (i.e., intensity of the cognitive symptoms), the challenge level must be considered. Indeed, two profiles appeared regarding the participants' musical level, with stress having a positive effect on the first-year students and a negative effect on the second-year students. Although the results have to be interpreted with caution because of the limited number of participants in each group (16 first-year students and 12 second-year students), they show that a high challenge level leads to a greater negative evolution of the perception of somatic symptoms between the two conditions. This could explain the differences between the two groups in this study and the contrasted findings of previous studies of the impact of stress on the quality of a music performance..$^{20,22,24,32,37}$

\section{Implications}

\section{Educational applications}

The ecological but controlled conditions of the present study have direct implications for music education. As interval precision and respect of the tonal center of a melody are linked to the intensity of cognitive symptoms, it would be interesting to focus on the prevention of these symptoms in solfeggio classes, for instance, by providing information about coping strategies in conservatories' education programs. More generally, a more distanced approach to the challenge would help to generate a positive effect of stress on music performance (i.e. as observed for the first-year students). The second-year students interpreted their somatic symptoms much more negatively than the first-year students and the results showed that stress had a negative effect on them. In addition to information on coping strategies, this study suggests that music institutions should organize 
examinations on a more regular basis in order to reduce the challenge level (and its negative effect) of the last year of the music program.

\section{Implications for singing research}

The present findings highlight the effect of stress - which can be positive or negative - on singing voice accuracy and thus the relevance of including emotional state among the multiple components required to sing in tune. ${ }^{8-10}$ In addition, we observed a dissociation between two criteria that are relevant in the evaluation of singing voice accuracy (i.e., interval deviation and tonal center deviation). ${ }^{7}$ In other words, this study indicates that emotional state and the dissociation between the vocal accuracy criteria should be taken into account in theoretical models explaining singing voice accuracy. Finally, the relationship between tempo and vocal accuracy, observed with both occasional singers ${ }^{2,3}$ and trained singers, ${ }^{4}$ is not visible when participants are instrumentalists (i.e., people who have a high level of musical knowledge but not much training in vocal production). Consequently, participants' music level should be taken into account in future investigations of singing voice accuracy.

\section{Conclusions}

By observing physiological, psychological and acoustical parameters in music students in stressful and non-stressful conditions, this study shows the effects of stress on singing voice accuracy. These effects can be either positive or negative, depending on the challenge level of the music examination. In addition to the pedagogical implications of these findings, this study provides relevant information about the singing voice accuracy process and the effects of stress on a sung performance. 


\section{References}

1. Berkowska M, Dalla Bella S. Reducing linguistic information enhances singing proficiency in occasional singers. Ann NY Acad Sci. 2009;1169:108-11.

2. Dalla Bella S, Berkowska M. Singing proficiency in the majority. Ann NY Acad Sci. 2009;1169(1):99-107.

3. Dalla Bella S, Giguère J-F, Peretz I. Singing proficiency in the general population. $J$ Acoust Soc Am. 2007;121(2):1182-9.

4. Larrouy-Maestri P, Morsomme D. Criteria and tools for objectively analysing the vocal accuracy of a popular song. Logoped Phoniatr Vocol. In press.

5. Pfordresher PQ, Brown S. Enhanced production and perception of musical pitch in tone language speakers. Atten Percep Psychophys. 2009;71(6):1385-98.

6. Pfordresher PQ, Brown S, Meier KM, Belyk M, Liotti M. Imprecise singing is widespread. J Acoust Soc Am. 2010;128(4):2182-90.

7. Larrouy-Maestri P, Lévêque Y, Schön D, Giovanni A, Morsomme D. The evaluation of singing voice accuracy: A comparison between subjective and objective methods. $J$ Voice. 2013;27(2):259e1-e5.

8. Dalla Bella S, Berkowska M, Sowinski J. Disorders of pitch production in tone deafness. Front Psychol. 2011;2:164.

9. Hutchins SM, Peretz I. A frog in your throat or in your ear? Searching for the causes of poor singing. J Exp Psychol Gen. 2012;141(1):76-97.

10. Pfordresher PQ, Brown S. Poor-pitch singing in the absence of "tone deafness". Music Percept. 2007;25(2):95-115.

11. Kenny, D. The Psychology of Music Performance Anxiety. Oxford University Press; 2011. 
12. Papageorgi I, Creech A, Welch G. Perceived performance anxiety in advances musicians specializing in different musical genres. Psychol Music. 2013;41(1):18-41.

13. Iusca D, Dafinoiu I. Performance anxiety and musical level of undergraduate students in exam situations: The role of gender and musical instrument. Procedia Soc Behav Sci. 2012;33:448-52.

14. Brodsky W. Music performance anxiety re-conceptualized: a critique of current research practices and findings. Med Probl Perform Art. 1996;11(3):88-98.

15. Papageorgi I, Hallam S, Welch GF. A conceptual framework for understanding musical performance anxiety. Res Stud Music Educ. 2007;28(1):83-107.

16. Salmon P. A psychological perspective on musical performance anxiety: A review of the literature. Med Probl Perform Art. 1990;5:2-11.

17. Fehm L, Schmidt K. Performance anxiety in gifted adolescent musicians. J Anxiety Disord. 2006;20(1):98-109.

18. Abel JL, Larkin KT. Anticipations of performance among musicians: physiological arousal, confidence, and state-anxiety. Psychol Music. 1990;18:171-82.

19. Brotons M. Effects of performing conditions on music performance anxiety and performance quality. J Music Ther. 1994;31:63-81.

20. Craske MG, Craig KD. Musical performance anxiety: The three systems-model and self-efficacy-theory. Behav Res Ther. 1984;22:267-80.

21. Fredrikson M, Gunnarsson R. Psychobiology of stage fright: the effect of public performance on neuroendocrine, cardiovascular and subjective reactions. Biol Psychol. 1992;33:51-61.

22. Hamann DL, Sobaje M. Anxiety and the college musician: a study of performance conditions and subject variables. Psychol Music. 1983;11:37-50. 
23. LeBlanc A, Chang Jin Y, Obert M, Siivola C. Effect of audience on music performance anxiety. J Res Music Educ. 1997;45(3):480-90.

24. Yoshie M, Kudo K, Ohtsuki T. Effects of psychological stress on state anxiety, electromyographic activity, and arpeggio performance in pianists. Med Probl Perform Art. 2008;23:120-32.

25. Yoshie M, Shigemasu K, Kudo K, Ohtsuki T. Effects of state anxiety on music performance: Relationship between the Revised Competitive State Anxiety Inventory 2 subscales and piano performance. Music Sci. 2008;8(1):55-84.

26. Bermudez de Alvear RM, Baron-Lopez FJ, Alguacil MD, Dawid-Milner MS. Interactions between voice fundamental frequency and cardiovascular parameters. Preliminary results and physiological mechanisms. Logoped Phoniatr Vocol. In press.

27. Giddens CL, Barron KW, Byrd-Craven J, Clark KF, Winter AS. Vocal indices of stress: A review. $J$ Voice. 2013;27:390 e21-e29.

28. Hagenaars MA, van Minnen A. The effect of fear on paralinguistic aspects of speech in patients with panic disorder with agoraphobia. J Anxiety Disord. 2005;19:521-37.

29. Ruiz R, Absil E, Harmegnies B, Legros C, Poch D. Time- and spectrum-related variabilities in stressed speech under laboratory and real conditions. Speech Commun. 1996;20:111-29.

30. Scherer KR. Effect of stress on fundamental frequency of the voice. J Acoust Soc Am. 1977;62:S25-S26.

31. Yerkes RM, Dodson JD. The relationship of strength of stimulus to rapidity of habit formation. J Comp Neurol Psychol. 1908;18:459-82. 
32. Yoshie M, Kudo K, Murakoshi T, Ohtsuki T. Music performance anxiety in skilled pianists: effects of social-evaluative performance situation on subjective, autonomic, and electromyographic reactions. Exp Brain Res. 2009;199(2):117-26.

33. Cox RH, Martens MP, Russell WD. Measuring anxiety in athletics: the revisited competitive state anxiety inventory-2. J Sport Exerc Psychol. 2003;25:519-33.

34. Martinent G, Ferrand C, Guillet E, Gautheur S. Validation of the French version of the Competitive State Anxiety Inventory - 2 Revised (CSAI-2R) including frequency and direction scales. Psychol Sport Exerc. 2010;11(1):51-7.

35. Elwood LS, Wolitzky-Taylor K, Olatunji BO. Measurement of anxious traits: A contemporary review and synthesis. Anxiety Stress Coping. 2012;25(6):647-66.

36. Rossi V, Pourtois G. Transient state-dependent fluctuations in anxiety measured using STAI, POMS, PANAS or VAS: A comparative review. Anxiety Stress Coping. 2012;25(6):603-45.

37. Kokotsaki D, Davidson JW. Investigating musical performance anxiety among music college singing students: A quantitative analysis. Music Educ Res. 2003;5(1):45-59. 


\section{Acknowledgments}

The authors thank the Centre Henri Pousseur in Liège, Guillaume Videlier, and David Magis for technical support. We also thank Céline Clijsters for her help with the data collection and the Royal Conservatories of Belgium for their collaboration. 


\section{Table and Figure Captions}

Table 1. Mean and standard error (in parentheses) of the stress measurements (heart rate, CSAI-2R questionnaire) for each music level (first and second), in the stressful and nonstressful conditions.

Table 2. Results of the Wilcoxon signed-rank test for the comparison between the stressful and non-stressful conditions, for each music level (first and second).

Table 3. Mean and standard error (in parentheses) for the changes in the stress measurements (heart rate, CSAI-2R questionnaire) between the non-stressful and the stressful conditions, for each music level (first and second). Differences between the two music levels were computed with the Mann-Whitney U test.

Figure 1. Illustration of the experimental procedure.

Figure 2. Musical score learned, recorded and analyzed. The melodic line is composed of different intervals from the second minor to the octave, with a tonal center particularly marked in F Major. The rhythm is composed of quarter notes and half notes (crotchets are considered as ornaments). Two indications are visible: the repeat and the tempo ( 80 beats per minute).

Figure 3. Illustration of the mean scores for the interval deviation criterion (in cents). The scores for the two music levels (first and second) are represented for the two conditions (stressful and non-stressful). Note that a low score represents a low deviation and thus a better accuracy. 
Figure 4. Illustration of the mean scores for the tonal center deviation criterion (in cents).

The scores for the two music levels (first and second) are represented for the two conditions (stressful and non-stressful). Note that a low score represents a low deviation and thus

better accuracy. 